\title{
Development of a New Scheme for the Solution of Initial Value Problems in Ordinary Differential Equations
}

\author{
${ }^{1}$ Ogunrinde R. B., ${ }^{2}$ Fadugba S. E. \\ ${ }^{1,2}$ Department of Mathematical Sciences, Ekiti State University, Ado Ekiti, Nigeria.
}

\begin{abstract}
This paper is on the development of a new scheme for the solution of initial value problems in ordinary differential equations. We present some basic concepts and fundamental theories which are very vital to the development of the scheme. The new scheme is of order seven and its derivation is based on the representation of the theoretical solution by perturbed polynomial function of degree four. This scheme is suitable for problems associated with the systems of ordinary differential equations having oscillatory or exponential solution.
\end{abstract}

Key words: Convergence, Exponential Solution, Ordinary Differential Equation,

\section{Introduction}

In the years past, a large number of methods suitable for solving ordinary differential equations have been proposed. A major impetus to developing numerical procedures was the invention of the calculus by Newton and Leibnitz as this led to accurate mathematical models for physical reality, such as Sciences, Engineering, Medicine and Business. These mathematical models cannot be usually solved explicitly and numerical method to obtain approximate solutions is needed. Another important aspect of the development of numerical methods was the creation of logarithms by Napier (1614) and others, giving a much simpler manner of carrying out the arithmetic operations of multiplication, division and exponentiation. Up to the late 1800's it appears that most mathematicians were quite broad in their interest. Generally, the efficiency of any of these methods depends on the stability and accuracy properties. The accuracy properties of different methods are usually compared by considering the order of convergence as well as the truncation error coefficient of the various methods. The sources of motivation for this work are those of [1] who proposed a numerical integration scheme of order four and [3] considered that of order five suited for the solution of initial value problem in ordinary differential equation of the form $F(x)=a_{0}+a_{1} x+a_{2} x^{2}+b\left(\right.$ reale $\left.e^{\rho x+\mu}\right)$ having exponential solution, where $a_{0}, a_{1}, a_{2}$ and $b$ are real undetermined coefficients, $\rho$ and $\mu$ are complex parameters and [6] introduced a new scheme of order six which is an improvement over [3]. There are many numerical integrating schemes to generate the numerical solutions developed by several authors such as [2], [4], [5] and [7] just to mention few. In this paper we shall consider the initial value problem of the form $y^{\prime}=f(x, y), y(a)=\eta$ and develop an algorithm of order seven which can effectively solve initial value problem in ordinary differential equation.

\section{Some Basic Concepts}

We shall consider the following basic concepts which are very vital to the development of the new scheme.

\subsection{Stability}

A numerical method is said to be stable if the difference between the numerical solution and the exact solution can be made as small as possible, that is if there exists two positive numbers $e_{0}$ and $K$ such that the following holds.

$\left\|y_{n}-y\left(x_{n}\right)\right\| \leq K\left\|e_{0}\right\|$

\subsection{Consistency [1]}

A numerical scheme with an increment function $\phi\left(x_{n}, y, h\right)$ is said to be consistent with the initial value problem under consideration, if

$\phi\left(x_{n}, y, h\right)=f(x, y)$

\subsection{Convergence}

A numerical method is said to be convergent if for all initial value problem satisfying the hypothesis of Lipschitz condition given by 
$\left|f(x, y)-f\left(x, y^{*}\right)\right| \leq L\left|y-y^{*}\right|$

Where the Lipschitz condition is denoted by $L$. The necessary and sufficient conditions for convergence are the stability and consistency.

\subsection{Round off Error}

This can be defined as the error due to computing device. They arise because it is possible to represent all real numbers exactly on a finite-state machine. It can be represented mathematically as

$R_{n+1}=y_{n+1}-p_{n+1}$

Where $y_{n+1}$ is the approximate solution and $p_{n+1}$ is the computer output. The magnitude depends in the storage and the arithmetic operation adopted. In such cases, double precision are employed to guarantee an adequate approximation.

\section{Development of a New Scheme of Order Seven}

This section presents the derivation of the new scheme of order seven for the solution of initial value problems in ordinary differential equations.

\subsection{The Basic Interpolant}

Let us assume that the theoretical solution $y(x)$ to the IVP problem

$y^{\prime}=f(x, y), y(a)=\eta$ can be represented locally in the interval $\left(x_{t}, x_{t+1}\right), t \geq 0$ by the polynomial interpolating function. [1]

$$
F(x)=a_{0}+a_{1} x+a_{2} x^{2}+a_{3} x^{3}+a_{4} x^{4}+b\left(\text { reale }{ }^{\rho x+\mu}\right)
$$

Where $a_{0}, a_{1}, a_{2}, a_{3}, a_{4}$ and $b$ are real undetermined coefficients, $\rho$ and $\mu$ are complex parameters.

If we put

$\rho=\rho_{1}+i \rho_{2}$

And $\rho=i \sigma=i^{2}=-1$ in (5), we obtain the following interpolating function

$F(x)=a_{0}+a_{1} x+a_{2} x^{2}+a_{3} x^{3}+a_{4} x^{4}+b e^{\rho_{1} x} \cos \left(\rho_{2} x+\sigma\right)$

If we move further to define the functions $R(x)$ and $\theta(x)$ as follows

$R(x)=e^{\rho_{1} x}$
$\theta(x)=\rho_{2} x+\sigma$

From (7) and (8), we obtain

$$
F(x)=a_{0}+a_{1} x+a_{2} x^{2}+a_{3} x^{3}+a_{4} x^{4}+b R(x) \cos (\theta x)
$$

We shall assume that $y_{t}$ is the numerical solution to the theoretical solution $y\left(x_{t}\right)$ and $f_{t}=f\left(x_{t}, y_{t}\right)$. We define mesh point as follows:

$x_{t}=a+t h$,

$t=0,1,2,3, \ldots$

We then move forward to impose the following constraints on the interpolating function (9).

a. That the interpolating function must coincide with the theoretical solution at $x=x_{t}$ and $x=x_{t+1}$. In order words, we required that

$F\left(x_{t}\right)=a_{0}+a_{1} x_{t}+a_{2} x^{2}{ }_{t}+a_{3} x^{3}{ }_{t}+a_{4} x^{4}{ }_{t}+b R\left(x_{t}\right) \cos \left(\theta x_{t}\right)$

i.e. $F\left(x_{t}\right)=y\left(x_{t}\right)$ and

$F\left(x_{t+1}\right)=a_{0}+a_{1} x_{t+1}+a_{2} x^{2}{ }_{t+1}+a_{3} x^{3}{ }_{t+1}+a_{4} x^{4}{ }_{t+1}+b R\left(x_{t+1}\right) \cos \left(\theta x_{t+1}\right)$

i.e. $F\left(x_{t+1}\right)=y\left(x_{t+1}\right)$.

b. We also require that the first, second, third, fourth and fifth derivatives with respect to $x$ of the interpolating function respectively coincide with the differential equation as well as its first, second, third, fourth and fifth derivatives with respect to $x_{t}$. In the other words, we require that: 


$$
\begin{aligned}
& F^{\prime}\left(x_{t}\right)=f_{t} \\
& F^{2}\left(x_{t}\right)=f_{t}^{1} \\
& F^{3}\left(x_{t}\right)=f_{t}^{2} \\
& F^{4}\left(x_{t}\right)=f_{t}^{3} \\
& F^{5}\left(x_{t}\right)=f_{t}^{4}
\end{aligned}
$$

This implies that

$$
\begin{aligned}
& F^{1}\left(x_{t}\right)=f_{t}=a_{1}+2 a_{2} x_{t}+3 a_{3} x_{t}^{2}+4 a_{4} x_{t}^{3}+b R\left(x_{t}\right)\left[\rho_{1} \cos \left(\theta x_{t}\right)-\rho_{2} \sin \left(\theta x_{t}\right)\right] \\
& f_{t}=a_{1}+2 a_{2} x_{t}+3 a_{3} x_{t}^{2}+4 a_{4} x_{t}^{3}+b R_{t}\left[\rho_{1} \cos \theta_{t}-\rho_{2} \sin \theta_{t}\right] \\
& F^{2}\left(x_{t}\right)=f_{t}^{1}=2 a_{2}+6 a_{3} x_{t}+12 a_{4} x_{t}^{2}+b R\left(x_{t}\right)\left[\left(\rho_{1}^{2}-\rho_{2}^{2}\right) \cos \left(\theta x_{t}\right)-2 \rho_{1} \rho_{2} \sin \left(\theta x_{t}\right)\right] \\
& f_{t}^{1}=2 a_{2}+6 a_{3} x_{t}+12 a_{4} x_{t}^{2}+b R_{t}\left[\left(\rho_{1}^{2}-\rho_{2}{ }^{2}\right) \cos \theta_{t}-2 \rho_{1} \rho_{2} \sin \theta_{t}\right] \\
& F^{3}\left(x_{t}\right)=f_{t}^{2}=6 a_{3}+24 a_{4} x_{t}+b R\left(x_{t}\right)\left[\left(\rho_{1}^{3}-3 \rho_{1} \rho_{2}^{2}\right) \cos \left(\theta x_{t}\right)+\left(\rho_{2}^{3}-3 \rho_{1}^{2} \rho_{2}\right) \sin \left(\theta x_{t}\right)\right] \\
& f_{t}^{2}=6 a_{3}+24 a_{4} x_{t}+b R\left(x_{t}\right)\left[\left(\rho_{1}^{3}-3 \rho_{1} \rho_{2}{ }^{2}\right) \cos \theta_{t}+\left(\rho_{2}{ }^{3}-3 \rho_{1}^{2} \rho_{2}\right) \sin \theta_{t}\right] \\
& F^{4}\left(x_{t}\right)=f_{t}^{3}=24 a_{4}+ \\
& b R\left(x_{t}\right)\left[\left(\rho_{1}^{4}+\rho_{2}{ }^{4}-6 \rho_{1}^{2} \rho_{2}{ }^{2}\right) \cos \left(\theta x_{t}\right)+\left(4 \rho_{1} \rho_{2}{ }^{3}-4 \rho_{1}^{3} \rho_{2}\right) \sin \left(\theta x_{t}\right)\right] \\
& f_{t}^{3}=24 a_{4}+b R_{t}\left[\left(\rho_{1}^{4}+\rho_{2}{ }^{4}-6 \rho_{1}^{2} \rho_{2}{ }^{2}\right) \cos \theta_{t}+\left(4 \rho_{1} \rho_{2}{ }^{3}-4 \rho_{1}^{3} \rho_{2}\right) \sin \theta_{t}\right] \\
& F^{5}\left(x_{t}\right)=f_{t}^{4}=b R\left(x_{t}\right)\left[\left(\rho_{1}{ }^{5}+5 \rho_{1} \rho_{2}{ }^{4}-10 \rho_{1}{ }^{3} \rho_{2}{ }^{2}\right) \cos \left(\theta x_{t}\right)+\left(10 \rho_{1}{ }^{2} \rho_{2}{ }^{3}-5 \rho_{1}{ }^{3} \rho_{2}\right) \sin \left(\theta x_{t}\right)\right] \\
& f_{t}^{4}=b R_{t}\left[\left(\rho_{1}^{5}+5 \rho_{1} \rho_{2}{ }^{4}-10 \rho_{1}^{3} \rho_{2}{ }^{2}\right) \cos \theta_{t}+\left(10 \rho_{1}^{2} \rho_{2}^{3}-5 \rho_{1}^{3} \rho_{2}\right) \sin \theta_{t}\right]
\end{aligned}
$$

Note that $F^{1}\left(x_{t}\right)=f_{t}, F^{2}\left(x_{t}\right)=f_{t}^{1}, F^{3}\left(x_{t}\right)=f_{t}^{2}, F^{4}\left(x_{t}\right)=f_{t}^{3}, F^{5}\left(x_{t}\right)=f_{t}^{4}$, where $f_{t}^{i t h}$ denotes the ith derivative of $(x, y)$ with respect to $x$ by setting $R_{t}=R\left(x_{t}\right)$ and $\theta_{t}=\theta\left(x_{t}\right)$. We obtain directly from (8) and (10) that

$$
\begin{aligned}
& R_{t+1}=R_{t} e^{\rho_{1} h} \\
& \theta_{t+1}=\theta_{t}+\rho_{2} h
\end{aligned}
$$

From (18a) we have

$$
b=\frac{f_{t}^{4}}{R_{t}\left[\left(\rho_{1}^{5}+5 \rho_{1} \rho_{2}{ }^{4}-10 \rho_{1}^{3} \rho_{2}{ }^{2}\right) \cos \theta_{t}+\left(10 \rho_{1}^{2} \rho_{2}^{3}-5 \rho_{1}^{4} \rho_{2}\right) \sin \theta_{t}\right]}
$$

From (17a)

$$
\begin{aligned}
& f_{t}^{3}=24 a_{4}+b R_{t}\left[\left(\rho_{1}{ }^{4}+\rho_{2}{ }^{4}-6 \rho_{1}{ }^{2} \rho_{2}{ }^{2}\right) \cos \theta_{t}+\left(4 \rho_{1} \rho_{2}{ }^{3}-4 \rho_{1}^{3} \rho_{2}\right) \sin \theta_{t}\right], \text { then } \\
& 24 a_{4}=f_{t}^{3}-b R_{t}\left[\left(\rho_{1}{ }^{4}+\rho_{2}{ }^{4}-6 \rho_{1}{ }^{2} \rho_{2}{ }^{2}\right) \cos \theta_{t}+\left(4 \rho_{1} \rho_{2}{ }^{3}-4 \rho_{1}{ }^{3} \rho_{2}\right) \sin \theta_{t}\right] \\
& a_{4}=\frac{1}{24}\left[f_{t}^{3}-\frac{\left[\left(\rho_{1}{ }^{4}+\rho_{2}{ }^{4}-6 \rho_{1}{ }^{2} \rho_{2}\right) \cos \theta_{t}+4\left(\rho_{1} \rho_{2}{ }^{3}-\rho_{1}{ }^{3} \rho_{2}\right) \sin \theta_{t}\right] f_{t}{ }^{4}}{\left[\left(\rho_{1}{ }^{5}+5 \rho_{1} \rho_{2}{ }^{4}-10 \rho_{1}{ }^{3} \rho_{2}{ }^{2}\right) \cos \theta_{t}+\left(10 \rho_{1}{ }^{2} \rho_{2}{ }^{3}-5 \rho_{1}{ }^{4} \rho_{2}\right) \sin \theta_{t}\right]}\right]
\end{aligned}
$$

From (16a)

$$
\begin{aligned}
& f_{t}^{2}=6 a_{3}+24 a_{4} x_{t}+b R\left(x_{t}\right)\left[\left(\rho_{1}^{3}-3 \rho_{1} \rho_{2}{ }^{2}\right) \cos \theta_{t}+\left(\rho_{2}{ }^{3}-3 \rho_{1}{ }^{2} \rho_{2}\right) \sin \theta_{t}\right], \text { where } \\
& x_{t}=(a+t h) \\
& a_{3}=\frac{1}{6}\left\{f_{t}^{2}-\left[f_{t}^{3}-\frac{\left[\left(\rho_{1}{ }^{4}+\rho_{2}{ }^{4}-6 \rho_{1}{ }^{2} \rho_{2}\right) \cos \theta_{t}+4\left(\rho_{1} \rho_{2}{ }^{3}-\rho_{1}{ }^{3} \rho_{2}\right) \sin \theta_{t}\right] f_{t}{ }^{4}}{\left[\left(\rho_{1}{ }^{5}+5 \rho_{1} \rho_{2}{ }^{4}-10 \rho_{1}{ }^{3} \rho_{2}{ }^{2}\right) \cos \theta_{t}+\left(10 \rho_{1}{ }^{2} \rho_{2}{ }^{3}-5 \rho_{1}{ }^{4} \rho_{2}\right) \sin \theta_{t}\right]}\right](a+t h)\right. \\
& \left.-\left[\frac{\left[\left(\rho_{1}+{ }^{4} \rho_{2}{ }^{2}-6 \rho_{1}{ }^{2} \rho_{2}{ }^{2}\right) \cos \theta_{t}+\left(\rho_{2}{ }^{3}-3 \rho_{1}{ }^{2} \rho_{2}\right) \sin \theta_{t}\right] f_{t}{ }^{4}}{\left[\left(\rho_{1}{ }^{5}+5 \rho_{1} \rho_{2}{ }^{4}-10 \rho_{1}{ }^{3} \rho_{2}{ }^{2}\right) \cos \theta_{t}+\left(10 \rho_{1}{ }^{2} \rho_{2}{ }^{3}-5 \rho_{1}{ }^{4} \rho_{2}\right) \sin \theta_{t}\right]}\right]\right\}
\end{aligned}
$$


From (15a)

$$
\begin{aligned}
& f_{t}^{1}=2 a_{2}+6 a_{3} x_{t}+12 a_{4} x_{t}{ }^{2}+b R_{t}\left[\left(\rho_{1}{ }^{2}-\rho_{2}{ }^{2}\right) \cos \theta_{t}-2 \rho_{1} \rho_{2} \sin \theta_{t}\right] \\
& 2 a_{2}=f_{t}{ }^{1}-6 a_{3} x_{t}+12 a_{4} x_{t}{ }^{2}+b R_{t}\left[\left(\rho_{1}{ }^{2}-\rho_{2}{ }^{2}\right) \cos \theta_{t}-2 \rho_{1} \rho_{2} \sin \theta_{t}\right]
\end{aligned}
$$

Then,

$$
\begin{aligned}
& a_{2}=\frac{1}{2}\left\{f_{t}^{1}-\left[f_{t}{ }^{2}-\left[f_{t}^{3}-\frac{\left[\left(\rho_{1}{ }^{4}+\rho_{2}{ }^{4}-6 \rho_{1}{ }^{2} \rho_{2}\right) \cos \theta_{t}+4\left(\rho_{1} \rho_{2}{ }^{3}-\rho_{1}{ }^{3} \rho_{2}\right) \sin \theta_{t}\right] f_{t}{ }^{4}}{\left[\left(\rho_{1}{ }^{5}+5 \rho_{1} \rho_{2}{ }^{4}-10 \rho_{1}{ }^{3} \rho_{2}{ }^{2}\right) \cos \theta_{t}+\left(10 \rho_{1}{ }^{2} \rho_{2}{ }^{3}-5 \rho_{1}{ }^{4} \rho_{2}\right) \sin \theta_{t}\right]}\right](a+t h)-\right.\right. \\
& \left.\frac{\left[\left(\rho_{1}^{3}-3 \rho_{1}{ }^{2} \rho_{2}{ }^{2}\right) \cos \theta_{t}+\left(\rho_{2}{ }^{3}-3 \rho_{1}{ }^{2} \rho_{2}\right) \sin \theta_{t}\right] f_{t}{ }^{4}}{\left[\left(\rho_{1}{ }^{5}+5 \rho_{1} \rho_{2}{ }^{4}-10 \rho_{1}{ }^{3} \rho_{2}{ }^{2}\right) \cos \theta_{t}+\left(10 \rho_{1}{ }^{2} \rho_{2}{ }^{3}-5 \rho_{1}{ }^{4} \rho_{2}\right) \sin \theta_{t}\right]}\right](a+t h) \\
& \left.-f_{t}^{3}-\frac{\left[\left(\rho_{1}{ }^{4}+\rho_{2}{ }^{4}-6 \rho_{1}{ }^{2} \rho_{2}\right) \cos \theta_{t}+4\left(\rho_{1} \rho_{2}{ }^{3}-\rho_{1}^{3} \rho_{2}\right) \sin \theta_{t}\right] f_{t}^{4}}{\left[\left(\rho_{1}^{5}+5 \rho_{1} \rho_{2}{ }^{4}-10 \rho_{1}{ }^{3} \rho_{2}{ }^{2}\right) \cos \theta_{t}+\left(10 \rho_{1}{ }^{2} \rho_{2}{ }^{3}-5 \rho_{1}{ }^{4} \rho_{2}\right) \sin \theta_{t}\right]}\right](a+t h)^{2}- \\
& \left.\left[\frac{\left[\left(\rho_{1}^{2}-\rho_{2}^{2}\right) \cos \theta_{t}-2 \rho_{1} \rho_{2} \sin \theta_{t}\right] f_{t}^{4}}{\left[\left(\rho_{1}^{5}+5 \rho_{1} \rho_{2}{ }^{4}-10 \rho_{1}^{3} \rho_{2}^{2}\right) \cos \theta_{t}+\left(10 \rho_{1}^{2} \rho_{2}^{3}-5 \rho_{1}^{4} \rho_{2}\right) \sin \theta_{t}\right]}\right]\right\}
\end{aligned}
$$

From (14a)

$$
\begin{aligned}
& f_{t}=a_{1}+2 a_{2} x_{t}+3 a_{3} x_{t}^{2}+4 a_{4} x_{t}^{3}+b R_{t}\left[\rho_{1} \cos \theta_{t}-\rho_{2} \sin \theta_{t}\right] \\
& a_{1}=f_{t}-2 a_{2} x_{t}+3 a_{3} x_{t}^{2}+4 a_{4} x_{t}^{3}+b R_{t}\left[\rho_{1} \cos \theta_{t}-\rho_{2} \sin \theta_{t}\right]
\end{aligned}
$$

Therefore,

$$
\begin{aligned}
& a_{1}=\left\{f_{t}-f_{t}^{1}-\left[f_{t}^{2}-\left[f_{t}^{3}-\frac{\left[\left(\rho_{1}^{4}+\rho_{2}{ }^{4}-6 \rho_{1}^{2} \rho_{2}\right) \cos \theta_{t}+4\left(\rho_{1} \rho_{2}^{3}-\rho_{1}^{3} \rho_{2}\right) \sin \theta_{t}\right] f_{t}^{4}}{\left[\left(\rho_{1}^{5}+5 \rho_{1} \rho_{2}{ }^{4}-10 \rho_{1}^{3} \rho_{2}{ }^{2}\right) \cos \theta_{t}+\left(10 \rho_{1}{ }^{2} \rho_{2}{ }^{3}-5 \rho_{1}{ }^{4} \rho_{2}\right) \sin \theta_{t}\right]}\right](a+t h)\right.\right. \\
& \left.-\frac{\left[\left(\rho_{1}^{3}-3 \rho_{1}{ }^{2} \rho_{2}{ }^{2}\right) \cos \theta_{t}+\left(\rho_{2}^{3}-3 \rho_{1}{ }^{2} \rho_{2}\right) \sin \theta_{t}\right] f_{t}^{4}}{\left[\left(\rho_{1}{ }^{5}+5 \rho_{1} \rho_{2}{ }^{4}-10 \rho_{1}^{3} \rho_{2}{ }^{2}\right) \cos \theta_{t}+\left(10 \rho_{1}{ }^{2} \rho_{2}^{3}-5 \rho_{1}^{4} \rho_{2}\right) \sin \theta_{t}\right]}\right](a+t h)^{2} \\
& -\frac{1}{2}\left[f_{t}^{2}-\left[f_{t}^{3}-\frac{\left[\left(\rho_{1}^{4}+\rho_{2}{ }^{4}-6 \rho_{1}{ }^{2} \rho_{2}\right) \cos \theta_{t}+4\left(\rho_{1} \rho_{2}{ }^{3}-\rho_{1}^{3} \rho_{2}\right) \sin \theta_{t}\right] f_{t}^{4}}{\left[\left(\rho_{1}{ }^{5}+5 \rho_{1} \rho_{2}{ }^{4}-10 \rho_{1}^{3} \rho_{2}{ }^{2}\right) \cos \theta_{t}+\left(10 \rho_{1}{ }^{2} \rho_{2}^{3}-5 \rho_{1}{ }^{4} \rho_{2}\right) \sin \theta_{t}\right]}\right](a+t h)-\right. \\
& \left.\left[\frac{\left[\left(\rho_{1}^{3}-3 \rho_{1} \rho_{2}{ }^{2}\right) \cos \theta_{t}+\left(\rho_{2}^{3}-3 \rho_{1}^{2} \rho_{2}\right) \sin \theta_{t}\right] f_{t}^{4}}{\left[\left(\rho_{1}{ }^{5}+5 \rho_{1} \rho_{2}{ }^{4}-10 \rho_{1}{ }^{3} \rho_{2}{ }^{2}\right) \cos \theta_{t}+\left(10 \rho_{1}{ }^{2} \rho_{2}{ }^{3}-5 \rho_{1}{ }^{4} \rho_{2}\right) \sin \theta_{t}\right]}\right](a+t h)^{2}-f_{t}^{3}\right\} \\
& -\left\{\frac{1}{6}\left[\frac{\left[\left(\rho_{1}{ }^{4}+\rho_{2}{ }^{4}-6 \rho_{1}{ }^{2} \rho_{2}\right) \cos \theta_{t}+4\left(\rho_{1} \rho_{2}{ }^{3}-\rho_{1}^{3} \rho_{2}\right) \sin \theta_{t}\right] f_{t}^{4}}{\left[\left(\rho_{1}{ }^{5}+5 \rho_{1} \rho_{2}{ }^{4}-10 \rho_{1}{ }^{3} \rho_{2}{ }^{2}\right) \cos \theta_{t}+\left(10 \rho_{1}{ }^{2} \rho_{2}{ }^{3}-5 \rho_{1}{ }^{4} \rho_{2}\right) \sin \theta_{t}\right]}\right](a+t h)^{3}\right. \\
& \left.+\frac{\left[\rho_{1} \cos \theta_{t}-\rho_{2} \sin \theta_{t}\right] f_{t}^{4}}{\left[\left(\rho_{1}^{5}+5 \rho_{1} \rho_{2}^{4}-10 \rho_{1}^{3} \rho_{2}^{2}\right) \cos \theta_{t}+\left(10 \rho_{1}^{2} \rho_{2}^{3}-5 \rho_{1}^{4} \rho_{2}\right) \sin \theta_{t}\right]}\right\}
\end{aligned}
$$

If we subtract (11) from (12) and apply (18), we obtain the following relations

$$
\begin{aligned}
y_{t+1}-y_{t}= & a_{0}+a_{1}\left(x_{t+1}-x_{t}\right)+a_{2}\left(x_{t+1}^{2}-x^{2}{ }_{t}\right)+a_{3}\left(x^{3}{ }_{t+1}-x_{t}{ }^{3}\right)+a_{4}\left(x_{t+1}^{4}-x_{t}^{4}\right) \\
& +b R_{t}\left[e^{\rho_{1} h}\left(\cos \theta_{t} \cos \rho_{2} h-\sin \theta_{t} \sin \rho_{2} h\right)\right]-\cos \theta_{t}
\end{aligned}
$$

Where,

$$
\begin{aligned}
& \left(x^{2}{ }_{t+1}-x^{2}{ }_{t}\right)=2 a h+(1+2 t) h^{2} \\
& \left(x^{3}{ }_{t+1}-x^{3}{ }_{t}\right)=3 a^{2} h+a h^{2}(3+6 t)+h^{3}\left(3 t^{2}+3 t+1\right) \\
& \left(x^{4}{ }_{t+1}-x^{4}{ }_{t}\right)=4 a^{3} h+6 a^{2} h^{2}(1+2 t)+4 a h^{3}\left(3 t^{2}+3 t+1\right)+h^{4}\left(4 t^{3}+6 t^{2}+4 t+1\right)
\end{aligned}
$$

To obtain the new scheme, putting the values of (20), (21), (22), (23), (26), (27) and (28) in (25), we have 


$$
\begin{aligned}
& y_{t+1}-y_{t}= \\
& a_{1}=\left\{f_{t}-f_{t}^{1}-\left[f_{t}^{2}-\left[f_{t}^{3}-\frac{\left[\left(\rho_{1}^{4}+\rho_{2}{ }^{4}-6 \rho_{1}^{2} \rho_{2}\right) \cos \theta_{t}+4\left(\rho_{1} \rho_{2}^{3}-\rho_{1}^{3} \rho_{2}\right) \sin \theta_{t}\right] f_{t}^{4}}{\left[\left(\rho_{1}^{5}+5 \rho_{1} \rho_{2}{ }^{4}-10 \rho_{1}^{3} \rho_{2}{ }^{2}\right) \cos \theta_{t}+\left(10 \rho_{1}{ }^{2} \rho_{2}^{3}-5 \rho_{1}^{4} \rho_{2}\right) \sin \theta_{t}\right]}\right](a+t h)-\right.\right. \\
& \left.\frac{\left[\left(\rho_{1}^{3}-3 \rho_{1}{ }^{2} \rho_{2}{ }^{2}\right) \cos \theta_{t}+\left(\rho_{2}{ }^{3}-3 \rho_{1}^{2} \rho_{2}\right) \sin \theta_{t}\right] f_{t}^{4}}{\left[\left(\rho_{1}{ }^{5}+5 \rho_{1} \rho_{2}{ }^{4}-10 \rho_{1}{ }^{3} \rho_{2}{ }^{2}\right) \cos \theta_{t}+\left(10 \rho_{1}{ }^{2} \rho_{2}{ }^{3}-5 \rho_{1}{ }^{4} \rho_{2}\right) \sin \theta_{t}\right]}\right](a+t h)^{2} \\
& -\frac{1}{2}\left[f_{t}^{2}-\left[f_{t}^{3}-\frac{\left[\left(\rho_{1}{ }^{4}+\rho_{2}{ }^{4}-6 \rho_{1}{ }^{2} \rho_{2}\right) \cos \theta_{t}+4\left(\rho_{1} \rho_{2}{ }^{3}-\rho_{1}{ }^{3} \rho_{2}\right) \sin \theta_{t}\right] f_{t}^{4}}{\left[\left(\rho_{1}{ }^{4}+5 \rho_{1} \rho_{2}{ }^{4}-10 \rho_{1}{ }^{3} \rho_{2}{ }^{2}\right) \cos \theta_{t}+\left(10 \rho_{1}{ }^{2} \rho_{2}{ }^{3}-5 \rho_{1}{ }^{4} \rho_{2}\right) \sin \theta_{t}\right]}\right](a+t h)-\right. \\
& \left.\left[\frac{\left[\left(\rho_{1}^{3}-3 \rho_{1} \rho_{2}{ }^{2}\right) \cos \theta_{t}+\left(\rho_{2}{ }^{3}-3 \rho_{1}{ }^{2} \rho_{2}\right) \sin \theta_{t}\right] f_{t}^{4}}{\left[\left(\rho_{1}{ }^{5}+5 \rho_{1} \rho_{2}{ }^{4}-10 \rho_{1}{ }^{3} \rho_{2}{ }^{2}\right) \cos \theta_{t}+\left(10 \rho_{1}{ }^{2} \rho_{2}{ }^{3}-5 \rho_{1}{ }^{4} \rho_{2}\right) \sin \theta_{t}\right]}\right](a+t h)^{2}-f_{t}^{3}\right\} h \\
& -\left\{\frac{1}{6}\left[\frac{\left[\left(\rho_{1}{ }^{4}+\rho_{2}{ }^{4}-6 \rho_{1}{ }^{2} \rho_{2}\right) \cos \theta_{t}+4\left(\rho_{1} \rho_{2}{ }^{3}-\rho_{1}{ }^{3} \rho_{2}\right) \sin \theta_{t}\right] f_{t}^{4}}{\left[\left(\rho_{1}{ }^{5}+5 \rho_{1} \rho_{2}{ }^{4}-10 \rho_{1}{ }^{3} \rho_{2}{ }^{2}\right) \cos \theta_{t}+\left(10 \rho_{1}{ }^{2} \rho_{2}{ }^{3}-5 \rho_{1}{ }^{4} \rho_{2}\right) \sin \theta_{t}\right]}\right](a+t h)^{3}\right. \\
& \left.+\frac{\left[\rho_{1} \cos \theta_{t}-\rho_{2} \sin \theta_{t}\right] f_{t}^{4}}{\left[\left(\rho_{1}^{5}+5 \rho_{1} \rho_{2}^{4}-10 \rho_{1}^{3} \rho_{2}^{2}\right) \cos \theta_{t}+\left(10 \rho_{1}^{2} \rho_{2}^{3}-5 \rho_{1}^{4} \rho_{2}\right) \sin \theta_{t}\right]}\right\} h \\
& +\frac{1}{2}\left\{f_{t}{ }^{1}-\left[f_{t}^{2}-\left[f_{t}^{3}-\frac{\left[\left(\rho_{1}{ }^{4}+\rho_{2}{ }^{4}-6 \rho_{1}{ }^{2} \rho_{2}\right) \cos \theta_{t}+4\left(\rho_{1} \rho_{2}{ }^{3}-\rho_{1}{ }^{3} \rho_{2}\right) \sin \theta_{t}\right] f_{t}^{4}}{\left[\left(\rho_{1}{ }^{4}+5 \rho_{1} \rho_{2}{ }^{4}-10 \rho_{1}{ }^{3} \rho_{2}{ }^{2}\right) \cos \theta_{t}+\left(10 \rho_{1}{ }^{2} \rho_{2}{ }^{3}-5 \rho_{1}{ }^{4} \rho_{2}\right) \sin \theta_{t}\right]}\right](a+t h)-\right.\right. \\
& \left.\frac{\left[\left(\rho_{1}^{3}-3 \rho_{1}^{2} \rho_{2}^{2}\right) \cos \theta_{t}+\left(\rho_{2}^{3}-3 \rho_{1}^{2} \rho_{2}\right) \sin \theta_{t}\right] f_{t}^{4}}{\left[\left(\rho_{1}{ }^{5}+5 \rho_{1} \rho_{2}{ }^{4}-10 \rho_{1}^{3} \rho_{2}{ }^{2}\right) \cos \theta_{t}+\left(10 \rho_{1}{ }^{2} \rho_{2}^{3}-5 \rho_{1}^{4} \rho_{2}\right) \sin \theta_{t}\right]}\right](a+t h) \\
& \left.-f_{t}^{3}-\frac{\left[\left(\rho_{1}^{4}+\rho_{2}{ }^{4}-6 \rho_{1}{ }^{2} \rho_{2}\right) \cos \theta_{t}+4\left(\rho_{1} \rho_{2}{ }^{3}-\rho_{1}{ }^{3} \rho_{2}\right) \sin \theta_{t}\right] f_{t}^{4}}{\left[\left(\rho_{1}{ }^{5}+5 \rho_{1} \rho_{2}{ }^{4}-10 \rho_{1}{ }^{3} \rho_{2}{ }^{2}\right) \cos \theta_{t}+\left(10 \rho_{1}{ }^{2} \rho_{2}{ }^{3}-5 \rho_{1}{ }^{4} \rho_{2}\right) \sin \theta_{t}\right]}\right](a+t h)^{2}- \\
& \left.\left[\frac{\left[\left(\rho_{1}{ }^{2}-\rho_{2}{ }^{2}\right) \cos \theta_{t}-2 \rho_{1} \rho_{2} \sin \theta_{t}\right] f_{t}^{4}}{\left[\left(\rho_{1}{ }^{5}+5 \rho_{1} \rho_{2}{ }^{4}-10 \rho_{1}{ }^{3} \rho_{2}{ }^{2}\right) \cos \theta_{t}+\left(10 \rho_{1}{ }^{2} \rho_{2}{ }^{3}-5 \rho_{1}{ }^{4} \rho_{2}\right) \sin \theta_{t}\right]}\right]\left(2 a h+(1+2 t) h^{2}\right)\right\} \\
& a_{3}=\frac{1}{6}\left\{f_{t}^{2}-\left[f_{t}^{3}-\frac{\left[\left(\rho_{1}{ }^{4}+\rho_{2}{ }^{4}-6 \rho_{1}{ }^{2} \rho_{2}\right) \cos \theta_{t}+4\left(\rho_{1} \rho_{2}{ }^{3}-\rho_{1}^{3} \rho_{2}\right) \sin \theta_{t}\right] f_{t}^{4}}{\left[\left(\rho_{1}{ }^{5}+5 \rho_{1} \rho_{2}{ }^{4}-10 \rho_{1}{ }^{3} \rho_{2}{ }^{2}\right) \cos \theta_{t}+\left(10 \rho_{1}{ }^{2} \rho_{2}{ }^{3}-5 \rho_{1}{ }^{4} \rho_{2}\right) \sin \theta_{t}\right]}\right](a+t h)-\right. \\
& \left.\left[\frac{\left[\left(\rho_{1}+{ }^{4} \rho_{2}{ }^{2}-6 \rho_{1}{ }^{2} \rho_{2}{ }^{2}\right) \cos \theta_{t}+\left(\rho_{2}{ }^{3}-3 \rho_{1}{ }^{2} \rho_{2}\right) \sin \theta_{t}\right] f_{t}{ }^{4}}{\left[\left(\rho_{1}{ }^{5}+5 \rho_{1} \rho_{2}{ }^{4}-10 \rho_{1}{ }^{3} \rho_{2}{ }^{2}\right) \cos \theta_{t}+\left(10 \rho_{1}{ }^{2} \rho_{2}{ }^{3}-5 \rho_{1}{ }^{4} \rho_{2}\right) \sin \theta_{t}\right]}\right]\right\}\left(3 a^{2} h+a h^{2}(3+6 t)+\right. \\
& \left.h^{3}\left(3 t^{2}+3 t+1\right)\right) \\
& \frac{1}{24}\left[f_{t}^{3}-\frac{\left[\left(\rho_{1}{ }^{4}+\rho_{2}{ }^{4}-6 \rho_{1}{ }^{2} \rho_{2}\right) \cos \theta_{t}+4\left(\rho_{1} \rho_{2}{ }^{3}-\rho_{1}{ }^{3} \rho_{2}\right) \sin \theta_{t}\right] f_{t}^{4}}{\left[\left(\rho_{1}{ }^{5}+5 \rho_{1} \rho_{2}{ }^{4}-10 \rho_{1}{ }^{3} \rho_{2}{ }^{2}\right) \cos \theta_{t}+\left(10 \rho_{1}{ }^{2} \rho_{2}{ }^{3}-5 \rho_{1}{ }^{4} \rho_{2}\right) \sin \theta_{t}\right]}\right]\left(4 a^{3} h+\right. \\
& \left.6 a^{2} h^{2}(1+2 t)+4 a h^{3}\left(3 t^{2}+3 t+1\right)+h^{4}\left(4 t^{3}+6 t^{2}+4 t+1\right)\right) \\
& +\frac{\left[e^{\rho_{1} h}\left(\cos \theta_{t} \cos \rho_{2} h-\sin \theta_{t} \sin \rho_{2} h\right)\right]-\cos \theta_{t} f_{t}{ }^{4}}{R_{t}\left[\left(\rho_{1}{ }^{5}+5 \rho_{1} \rho_{2}{ }^{4}-10 \rho_{1}{ }^{3} \rho_{2}{ }^{2}\right) \cos \theta_{t}+\left(10 \rho_{1}{ }^{2} \rho_{2}{ }^{3}-5 \rho_{1}{ }^{4} \rho_{2}\right) \sin \theta_{t}\right]}
\end{aligned}
$$

Equation (29) is the new scheme of order seven. 


\section{Conclusion}

This paper considers the development of a new numerical scheme for the solution of initial value problem in ordinary differential equations. After a successful derivation of the algorithm, it is obvious that the scheme will be useful in solving initial value problem most especially problems having oscillatory or exponential solutions.

Comparing the scheme with the existing standard schemes especially that of (Ibijola 1997) which is of order Five, (Ogunrinde 2010) which is of order Six, in that ours is of order seven which shows that it is of higher order.Owing to time and computer time allocation, we have not considered the analysis of stability property of this scheme in great details.

\section{References}

[1] Fatunla S. O. (1980): "Numerical Integrators for Stiff and Highly Oscillatory Differential Equations", Mathematics of Computation 34, 373-390.

[2] Gautschi W. (1961): "Numerical Integration of Ordinary Differential Equations based on Trigonometric Polynomials", Numerische Mathematik 3, 381-397.

[3] Ibijola E. A. (1997): New Schemes for Numerical Integration of Special Initial Value Problems in Ordinary Differential Equations Ph.D. Thesis, University of Benin, Nigeria.

[4] Ibijola E. A and Ogunrinde R. B. (2010): “On a New Numerical Scheme for the Solution of Initial Value Problems",Accepted Australian Journal of Basic and Applied Sciences.

[5] Joseph-Loius Lagrange. (1813): "On Root finding and Polynomial Interpolation in Ordinary Differential Equations".

[6] Ogunrinde R. B. (2009): On a New Numerical Scheme for the Solution of Initial Value Problem in Ordinary Differential Equation s.

[7] Wallace C. S and Gupta G. K. (1973): "General Linear Multistep Methods to Solve Ordinary Differential Equations". The Australian Computer Journal, Vol. 5, 62-69. 